\title{
Archipel
}

ARCHIPEL Études interdisciplinaires sur le monde insulindien

$98 \mid 2019$

Varia

\section{Tony Reid, Mataram : A novel of love, faith and power in early Java}

\section{Henri Chambert-Loir}

\section{(2) OpenEdition \\ 1 Journals}

\section{Édition électronique}

URL : http://journals.openedition.org/archipel/1493

DOI : 10.4000/archipel.1493

ISSN : 2104-3655

\section{Éditeur}

Association Archipel

\section{Édition imprimée}

Date de publication : 3 décembre 2019

Pagination : 266-268

ISBN : 978-2-910513-82-5

ISSN : 0044-8613

\section{Référence électronique}

Henri Chambert-Loir, «Tony Reid, Mataram : A novel of love, faith and power in early Java », Archipel [En ligne], 98 | 2019, mis en ligne le 12 décembre 2019, consulté le 18 mars 2021. URL : http:// journals.openedition.org/archipel/1493; DOI : https://doi.org/10.4000/archipel.1493 
de lire le monde - littéralement : en déchiffrant les mots qui l'expriment. Les mots sont emboîtés les uns dans les autres («Batur, sebagai sistem kebahasaan, minimal adalah akronim, atau wancahan, atau jarwa-dhosok, yaitu pendekan dari mbat-mbatananing tutur, atau badaning catur. ", 213), ils riment et ils résonnent. Leurs multiples correspondances tracent dans l'univers des hommes des lignes de signification. Ce discours englobe le macrocosme et le monde invisible. Il montre que les actions humaines (les constructions sociales, les arts) sont dictées par l'environnement. On frôle parfois le jeu de mots ou le jeu tout court (« Karna saya ini senengnya ngomong ngalor-ngidul yang gak masuk di nalar. », 212).

L'invention d'une sémiologie javanaise, peut-être, et une façon originale d'aborder une micro-région d'Indonésie, son environnement, ses habitants et sa culture.

L'illustration de couverture est faite d'un dessin (par l'auteur lui-même probablement) de deux personnages masqués, Bancak et Dhoyok, qui sont deux incarnations antagonistes du peuple (voir la couverture du présent volume), tandis que le dos de l'ouvrage s'orne du dessin d'un acteur de Reog Grogol Bejiharjo.

Henri Chambert-Loir

\section{Littératures}

Tony Reid, Mataram : A novel of love, faith and power in early Java. Monsoon (UK), 2018, 336 p., ISBN : 9781912049127.

C'est assurément une surprise d'apprendre qu'Anthony Reid, éminent historien de l'Asie du Sud-Est, s'est offert le plaisir, à l'âge de la retraite, d'écrire un roman. On peut concevoir qu'un historien désire investir les personnages historiques qu'il a fréquentés toute sa vie de sentiments et d'émotions tangibles, ou bien qu'il désire combler certains vides des archives par la mise en scène d'hypothèses plausibles, ou encore que, par le biais d'une fiction, il désire exprimer une opinion personnelle sur des événements qu'il a observés jusque là « objectivement ». Mais foin de tout cela, deuxième surprise, Reid a écrit un roman d'aventures, avec un héros, quelques âmes valeureuses et des méchants par légions, avec un despote oriental, des coups de théâtre, des ruines, la jungle, un tigre, une éruption volcanique, des fêtes, des batailles et même un brin d'érotisme. La signature (Tony Reid) est là pour nous prévenir : l'auteur est le savant dans l'intimité.

Nous ne sommes pas loin des sources pour autant. Reid-romancier ne cite pas les grands noms de l'histoire de l'époque (le tout début du $17^{\mathrm{e}} \mathrm{s}$.), mais ce roman échevelé tire parti des sources historiques que Reid-savant connaît par coeur. Les historiens reconnaîtront ici et là des personnages (le très bizarre 
portrait d'Edmund Scott, par exemple), des faits ou des documents.

En octobre 1608, Thomas Hodges, commis de la East India Company arrivé à Banten avec la petite flotte commandée par William Keeling, descend à terre et négocie l'achat de poivre pour son commandant malade. Il se familiarise d'abord avec les tavernes et les prostituées pour le compte des matelots, mais très vite avec des aspects autrement significatifs de la culture locale : sur invitation de la reine-mère, il assiste à une série de rites destinés à entrer en contact avec l'âme d'une défunte : un spectacle masqué, une danse de possession, puis la transe d'une vieille magicienne (balian), qui entre en contact avec la défunte et incidemment révèle des faits de la vie privée de Hodges. La reine elle-même affirme qu'elle est musulmane en même temps que javanaise dans sa foi. Hodges tente vainement d'expliquer sa propre religion et certaines coutumes anglaises, mais il parvient à attirer l'attention lorsqu'il explique le fonctionnement d'une lunette marine, d'une montre et de la notation musicale.

Il fait très rapidement la connaissance de Sri, fille d'un grand marchand de la ville. Elle parle portugais et lui servira de traductrice tout au long de l'histoire. Elle sera aussi sa femme et la compagne de ses tribulations. C'est pour elle, initialement, qu'il décide de rester à Java, au lieu de repartir pour l'Europe avec sa flotte chargée d'épices, mais il conçoit bientôt le plan ambitieux d'être le premier marchand européen à Mataram et de gagner le marché de Java à la compagnie anglaise.

Les aventures s'enchaînent sans discontinuer, au prix de quelques invraisemblances, et le conduisent à Jepara, Kudus et Mataram. Hodges fabrique une fausse lettre de King James au Panembahan Mataram; il prend à son service un jeune Chinois dégourdi, qui, lui aussi, veut être le premier commerçant à Mataram. Il est fait prisonnier par des fanatiques musulmans, se convertit à l'islam sous les coups, est libéré par des Javanais bienveillants et parvient à Mataram, où se trouve déjà un sage-magicien polyglotte occidental conquis aux moeurs javanaises : Romo, un Jésuite originaire d'Angleterre, papiste adepte de l'inculturation, une sorte de Matteo Ricci javanisé. Hodges et lui se disputent sur des questions de dogme, mais s'entendent sur des sujets scientifiques et techniques; c'est grâce à lui que Hodges rencontre Pangeran Anggalaga, fils du Panembahan et futur Sultan Agung. Sous le règne du père comme du fils, Hodges est mis à profit pour ses connaissances en artillerie ; il participe même à des expéditions contre Pajang et Surabaya. Mais il est sommé de devenir Javanais, or c'est une notion dont la définition est en pleine mutation. Pangeran Anggalaga, qui se voit déjà roi, confie son éducation à un kyiai syncrétiste. Celui-ci emmène Hodges sur trois sites censés représenter la quintescence de la spiritualité javanaise : Borobudur, « Brambanan » et Bayat, qui représente la fusion des religions anciennes et de l'islam.

Sri, entretemps, met au monde un garçon. L'enfant est baptisé en même temps qu'il est l'objet d'un rite javanais. Romo pousse le laxisme jusqu'à célébrer le 
mariage de Hodges et Sri, alors qu'il a déjà une femme en Angleterre. Mais tout ceci ne peut que se terminer très mal. Le dernier chapitre est un épilogue : le fils a près de dix ans, il a un petit frère, il va être dûment circoncis. Il appert que Hodges s'est déclaré musulman et qu'il est mort quelque temps plus tard, sur un champ de bataille, peut-être assassiné sur ordre du roi.

Le roman est l'histoire d'un échec sur tous les plans : love (Sri épouse l'homme dont Hodges était jaloux), faith (Hodges renie sa foi et se convertit à l'islam), power (Hodges n'obtient rien de l'accord politique et commercial qu'il ambitionnait). Cet échec n'est pas explicitement formulé ; le roman n'est pas une tragédie ; le paragraphe final attribue au fils de Hodges, de poursuivre la mission de son père - mais l'échec est patent et il correspond, sur le plan romanesque, à une vérité historique : les Anglais ont échoué, place aux Hollandais.

Le livre ne montre aucun effort de style : aucune envolée lyrique sur les affres de l'amour ou les beautés de la nature, aucune préciosité d'expression ou de classicisme, nous sommes dans un roman d'action. Un roman, cependant, où l'on pense et l'on parle énormément. La préoccupation des Européens (Portugais, Hollandais, Anglais) est le commerce, qui s'effectue dans un climat de violence, car ils transportent en Asie leurs querelles européennes. Ils apportent à Java leurs connaissances en artillerie et des produits des technologies nouvelles (des longue-vue, des lorgnons, des horloges, des instruments de musique...), mais aucune science. Ils sont grossiers, brutaux et turbulents, mais ils ne sont pas encore une menace.

La préoccupation des Javanais, par contre, est la religion ; d'un point de vue spirituel d'abord, mais aussi politique. Le Panembahan Mataram organise un débat - comme l'avait fait le sultan moghol Akbar à sa cour de Fatehpur Sikri, trente ans plus tôt - entre les représentants de quatre religions : islam (un kiai), catholicisme (Romo), protestantisme (Hodges) et javanisme (un ascète), qui exposent les mérites de leur religion respective et leurs avantages sur les autres, ainsi que la place qu'y tient le roi. Le Panembahan est évidemment séduit par l'idée que le roi d'Angleterre est chef de l'Église, alors que l'islam regarde le souverain comme un simple mortel. Hodges, du début à la fin, est le catholique buté qui sait détenir la vérité et tient toute autre croyance pour hérésie ou superstition. Les religions venues d'Occident, cependant, l'islam comme le chritianisme, ont rendu plus aigüe encore la question de la quête de la vérité ultime : sont-elles aussi, à côté de la religion locale et de celles de l'Inde et de la Chine, de nouvelles voies vers Dieu?

Ainsi que le formule la reine de Banten : "Il n'y a qu'une vérité, mais dieu seul la connait. Ce pour quoi les hommes se battent ne sont que les noms, les mots qu'ils utilisent à son propos. Mais ces mots ne peuvent être dieu. Je ne peux le trouver qu'en gardant le silence et en écoutant ce qui est en moi. »

Ainsi va le roman. Du point de vue de l'Histoire, on connaît la suite.

Henri Chambert-Loir 